\title{
Robotic Eddy Current Thermography: Simulations and experiments
}

\author{
By Y. Mokhtari*, C Ibarra-Castanedo*, P. Servais ${ }^{* *}$ and X Maldague* \\ *Department of electrical and computer engineering, LAVAL University, Quebec city, Canada, \\ E-mail:mohammed-yacine.mokhtari.1@ulaval.ca,xavier.maldague@gel.ulaval.ca \\ ** Level 3 Eddy Current and Infrared Testing, NDT Pro, Libramont, Belgium, Email: pierreservais@skynet.be
}

\section{Acknowledgment}

This research was supported by both governments of Wallonia/Brussels and Quebec, and by the Natural Sciences and Engineering Research Council of Canada (NSERC). We are also thankful to the Wallonie Aerotraining Network (WAN) for the provided expertise. We also thank Visioo-Image Ltd. for the technical help.

\begin{abstract}
Eddy current thermography (ECT) is an active thermography method. The inductive excitation generates Eddy currents in an electrically-conductive specimen. In presence of defects, the eddy current flow is affected by these discontinuities leading to changes in the temperature distribution in the specimen around the defects. These changes are observed by an infrared camera. In this paper, we present a robotic application of the method. Simulations are performed using $\mathrm{COMSOL}^{\circledR}$ Multiphysics by varying different parameters. Experiment is realised on an aluminum plate with cracks of different sizes created artificially in our laboratory. The resulting images are reconstructed with a dedicated algorithm.
\end{abstract}

Keywords: Eddy current thermography, nondestructive testing (NDT), Robotic NDT, 3D FEM.

\section{Introduction}

Over the last decades, thermographic Nondestructive testing (NDT) has been largely used in the aerospace industry. One of the main advantages of the technique is to inspect a relatively large area in a short time. Thermographic NDT can be divided in two categories: passive and active. Passive thermography doesn't need an external heat source and is used to test specimen that are naturally at different temperature than the ambient. Active thermography uses an external heat source to excite thermally the inspected material and observe changes in the temperature distribution.

Eddy current thermography (ECT) is an active thermography method. The inductive excitation generates, thanks to the electromagnetic force (Lenz-Faraday's Law), a circular current (Eddy current) [2], [4] in an electricallyconductive specimen that is inspected [1]. This current heats the specimen due to the resistance of the specimen (Joules losses). The presence of a discontinuity (a defect) then affects the temperature distribution in the specimen because of the perturbation of the current flow in the material. These changes are finally captured by an infrared camera. One of the most important parameter to take in account in Eddy current testing is the Skin Depth. It represents the maximum depth at which the current will "penetrate" the specimen. The Skin Depth depends on the frequency of excitation, the magnetic permeability, and the resistivity of the material [1], [2], [4].

Improvements in IR cameras have led to several researches in Eddy current thermography topic. In [1], inspection is deployed with an $X-Y$ table. Simulations have been done on different specimens. It was shown that 3D simulations give better visualizations of the defects. Results have shown the capability of the method and a crack detection limit has been established. However, the resulting images were not processed. Also, the inspection could not proceed on complex shaped specimens (Curvatures).

In this work, Eddy current thermography is performed using a 6 degree of freedom (Dof) robotic arm. Simulations are achieved with $\mathrm{COMSOL}^{\circledR}$ Multiphysics. An aluminum plate with defects (line slots modelling cracks) with different size is modeled in software. In the experiments, an aluminum plate with defects (line slots) created artificially in our laboratory is inspected. The inspection is achieved with a 6 axis robotic arm. Resulting image is reconstructed using an algorithm developed in [6]. 


\subsection{1/qirt.2016.091}

\section{Simulation with $\mathrm{COMSOL}^{\circledR}$ Multiphysics}

$\mathrm{COMSOL}^{\circledR}$ Multiphysics is finite element modeling software for numerical simulations enabling to model physical principles. To obtain a solution for Eddy current thermography, it is necessary to solve the magnetic propagation simultaneously with the heat transfer equations. Equations for the simulation model are given as follows [3]:

$$
\rho C_{p} \frac{\partial T}{\partial t}-\nabla \cdot k \nabla T=Q
$$

Where $\rho\left(\mathrm{kg} / \mathrm{m}^{3}\right)$ is the density, $C_{p}(J / K)$ is the specific heat, $k(W /(m . K))$ is the thermal conductivity, and $Q$ is the inductive heating.

$$
Q=\frac{1}{\sigma}\left|J_{s}\right|^{2}=\frac{1}{\sigma}|\sigma E|^{2}
$$

with $E$, the electric field intensity vector, $J_{S}$ the current density, and $\sigma\left(S . m^{-1}\right)$ the electrical conductivity which is temperature dependent as it is shown in the following equation:

$$
\sigma=\frac{\sigma}{1+\alpha\left(T-T_{0}\right)}
$$

With $\sigma_{0}$ is the conductivity at the reference temperature $T_{0}$, and $\alpha(\Omega . m)$ is the coefficient of resistivity.

Simulations are done in 3D since this gives better visualizations of defects [1]. Simulations are achieved on an aluminium plate with artificial defects created in our laboratory (line slots modeling cracks). The following parameters were varied: defects size $(3$ defects of size respectively: width $=$ depth $=0.2 \mathrm{~mm}$; width $=$ depth $=0.5$; all defects have a length of $50 \mathrm{~mm}$ (specimen's width)), frequency of excitation, and position of the coil (parameters are discussed below). Other parameters will be studied in future work to optimize results such as the number of turns for the coil and current circulating in it, lateral velocity and lift-off over the specimen. The following table shows the main simulations parameters:

Table 1: Simulations parameters

\begin{tabular}{|c|c|c|c|}
\hline Parameters & Values & Parameters & Values \\
\hline Current & $15 \mathrm{~A}$ & $\begin{array}{c}\text { Distance between the coil } \\
\text { and the plate (lift-off) }\end{array}$ & $3 \mathrm{~mm}$ \\
\hline Number of turns for the coil & 10 & Time of heating & $1 \mathrm{~s}$ \\
\hline Radius of the wire of the coil & $1 \mathrm{~mm}$ & Material of the coil & Copper \\
\hline Radius of the coil & $3 \mathrm{~mm}$ & Material of the specimen & Aluminum \\
\hline
\end{tabular}

\subsection{Simulations results}

\subsection{1 $\mathrm{f}=100 \mathrm{KHz}$ (excitation frequency), $\mathrm{T}=1 \mathrm{~s}$ (time of heating), $\mathrm{D}=3 \mathrm{~mm}$ (lift-off)}

The following figure shows simulations results with the previous parameters:
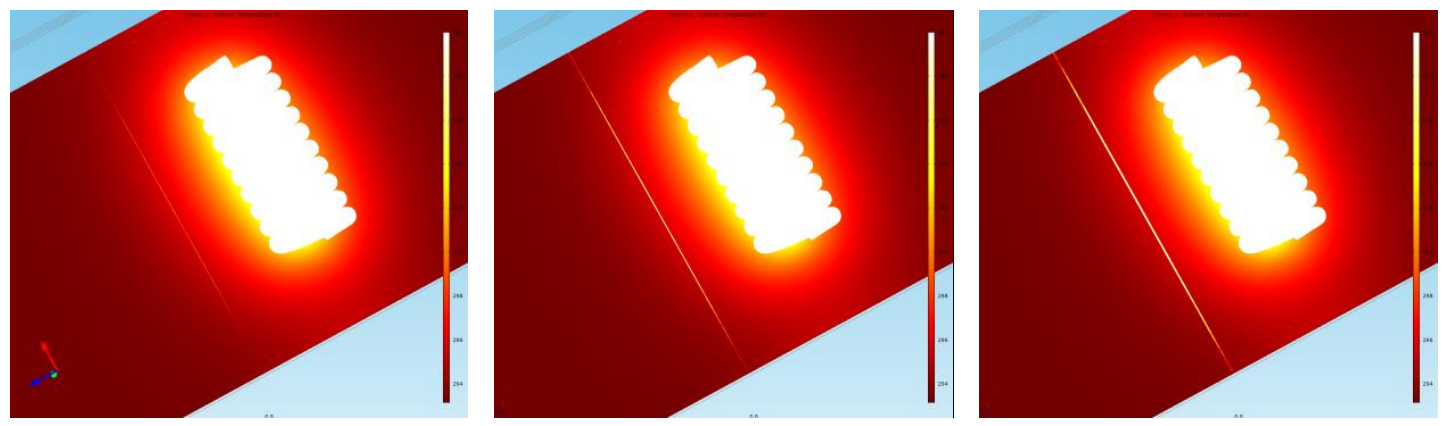

Fig. 1. Simulations Results with $f=100 \mathrm{KHz}$ and $T=1 \mathrm{~s}$ (heating time): left (defect $w$ (width) $=0.2 \mathrm{~mm}, d$ (depth) $=0.2 \mathrm{~mm}$ ), Middle (defect $w=0.5 \mathrm{~mm}, d=0.5 \mathrm{~mm}$ ), right (defect $w=1 \mathrm{~mm}, d=1 \mathrm{~mm}$ ). 
2.1.2 $\mathrm{f}=200 \mathrm{KHz}$ (excitation frequency), $\mathrm{T}=1 \mathrm{~s}$ (time of heating), $\mathrm{D}=3 \mathrm{~mm}$ (lift-off)
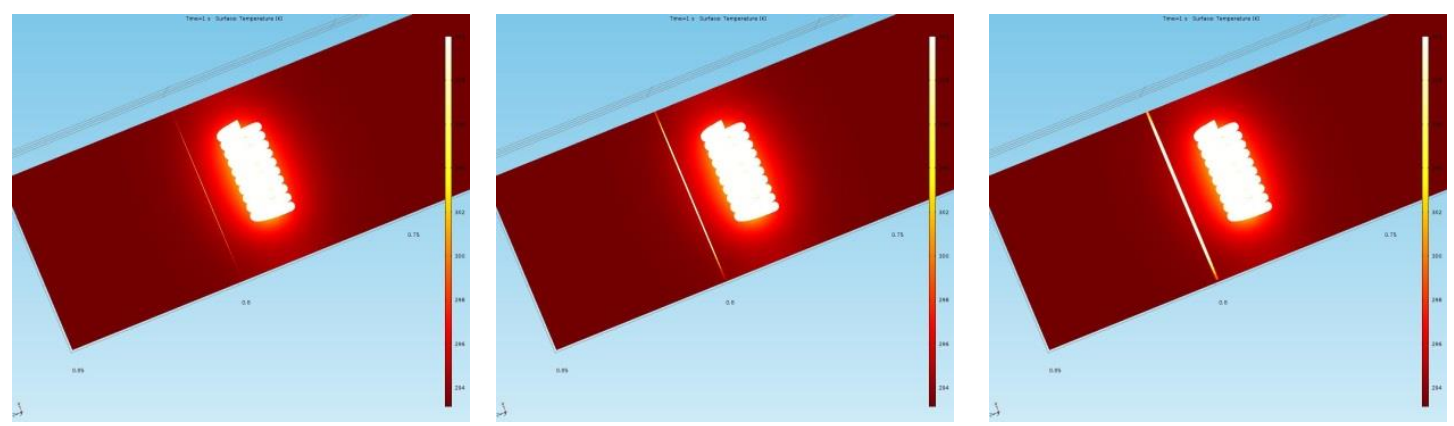

Fig. 2. Simulations Results with $f=200 \mathrm{KHz}$ and $T=1 \mathrm{~s}$ (heating time): left (defect $w$ (width) $=0.2 \mathrm{~mm}, d$ (depth) $=0.2 \mathrm{~mm}$ ), Middle (defect $w=0.5 \mathrm{~mm}, d=0.5 \mathrm{~mm})$, right (defect $w=1 \mathrm{~mm}, d=1 \mathrm{~mm}$ ).

From the figure, defects are very well detected, even the smallest one $(0.2 \mathrm{~mm})$.

\subsection{3 $\mathrm{f}=200 \mathrm{KHz}$ (excitation frequency), $\mathrm{T}=1 \mathrm{~s}$ (time of heating), $\mathrm{D}=7 \mathrm{~mm}$ (lift-off)}
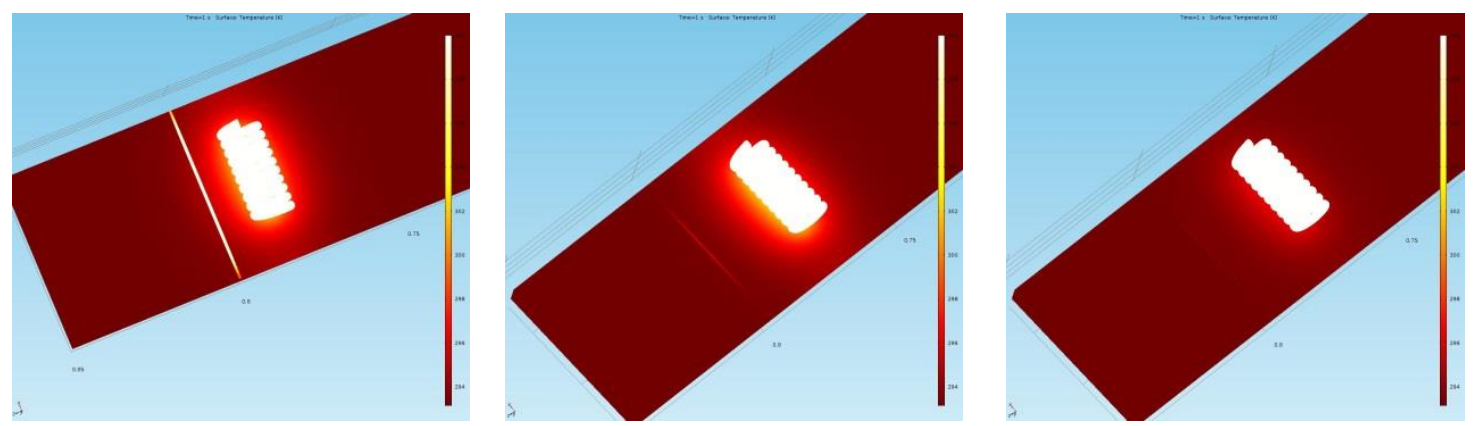

Fig. 3. Simulations Results with $f=200 \mathrm{KHz}$, defect $w=1 \mathrm{~mm}, d=1 \mathrm{~mm}$, and $T=1 \mathrm{~s}$ (heating time): left (coil position $P=$ $0.5 \mathrm{~cm}$ far from the defect, lift-off $=3 \mathrm{~mm})$, Middle $(P=2.5 \mathrm{~cm}$, lift-off $=3 \mathrm{~mm})$, right $(P=2.5 \mathrm{~cm}$, lift-off $=7 \mathrm{~mm})$.

Based on results obtained, we can see in figure 1, the three defects are clearly detected. Better results are obtained when using the frequency of excitation $f=200 \mathrm{KHz}$ (figure 2) which is the frequency used when performing experimental Eddy current inspection of an aluminum alloy specimen in the laboratory. In the last simulation, the position of the coil is varied (lift-off and horizontal distance from the defect). From the results, it is observed that when the lift-off or the horizontal distance of the coil from the defect is relatively large, the magnetic field around the defect is less important. This leads to a decrease in Eddy current's amplitude around the defect and the heating is not as important (figure 3 middle and right) as if the coil is close to the defect (figure 3 left). Consequently, in experiments a frequency of $f=200 \mathrm{KHz}$ and a lift-off of $3 \mathrm{~mm}$ will be used.

\section{Experimental results}

\subsection{Experimental setup}

The experiment is performed on an aluminum plate (aluminum alloy 7075) with two defects created artificially in our laboratory (line slots modelling cracks), experiment parameters as in Table 1. The current in the coil is $I=10 \mathrm{~A}$. Other coil parameters (material, number of turns...etc.) are detailed in table 1. Defects sizes are, respectively, width $=0.5,1 \mathrm{~mm}$, depth $=0.5,1 \mathrm{~mm}$, and length $=70 \mathrm{~mm}$ (width of the specimen). Eddy current thermography is deployed with 6 axis robot FANUC LrMate 200iD/7L (figure 4). The whole specimen is then scanned with the robot while being heated with the coil. The results are observed with an infrared camera FLIR A65. Pseudostatic matrix reconstruction [6] is performed on the resulting images to have an image of the plate with all defects visible on it. Principal component thermography (PCT) algorithm [7] is applied to the results of the reconstruction (figure 8). 


\subsection{1/qirt.2016.091}

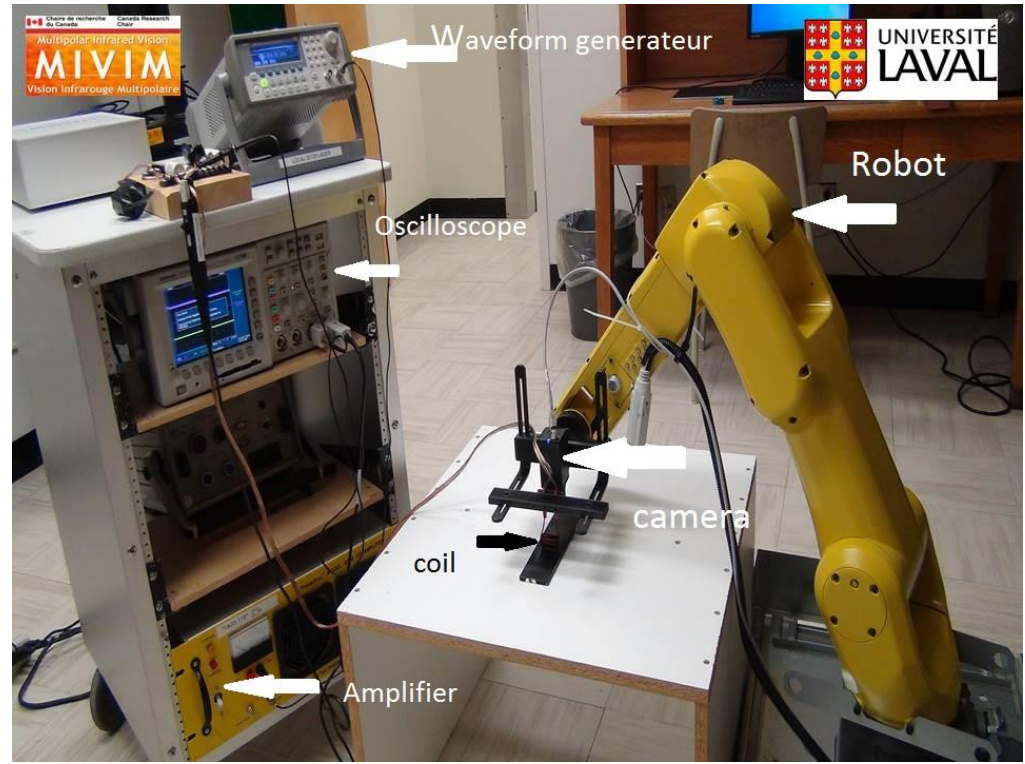

Fig. 4 Experimental setup

The eddy current equipment is:

- Coil (characteristics in table-1)

- Amplifier: Industrial Test Equipment "PowerTron" Model 500A RF

- Waveform generator: Agilent 33220A 200MHz

- An oscilloscope to monitor the excitation current (fig. 3): Tektronix TDS 3034B.

\subsection{Results}

The experiment is performed with a frequency of excitation $f=200 \mathrm{KHz}$, the lift-off $D=3 \mathrm{~mm}$, and the speed of inspection is $V=10 \mathrm{~mm} / \mathrm{s}$. Results are shown in the following figures:
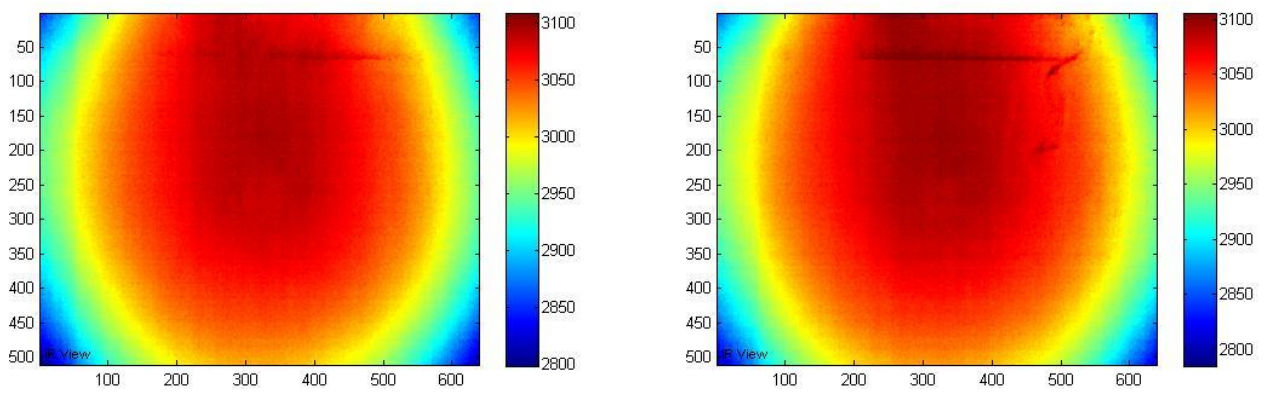

Fig. 5 Experimental results on the aluminum plate: defect's width and depth is $0.5 \mathrm{~mm}$ (left), defect's width and depth is $1 \mathrm{~mm}$ (right). In both cases, defect length is $50 \mathrm{~mm}$ (specimen width). 

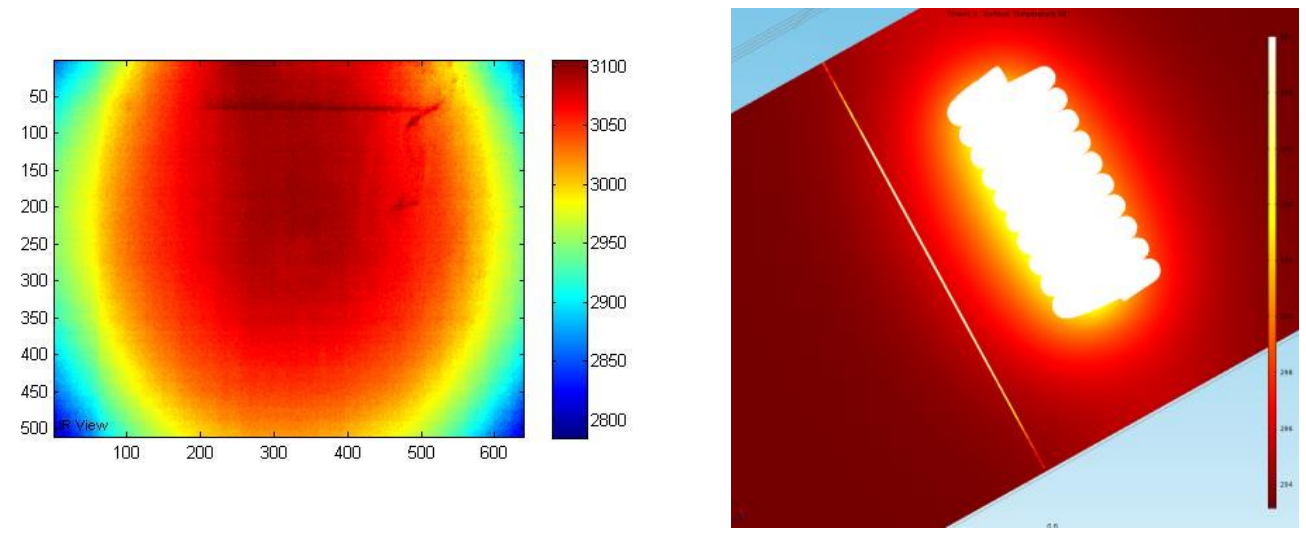

Fig. 6 Experimental results on the aluminum plate for the defect of $1 \mathrm{~mm}$ (left), orientation is $90^{\circ}$ with respect to simulations (right): line slot corresponding to defect is visible on the top.

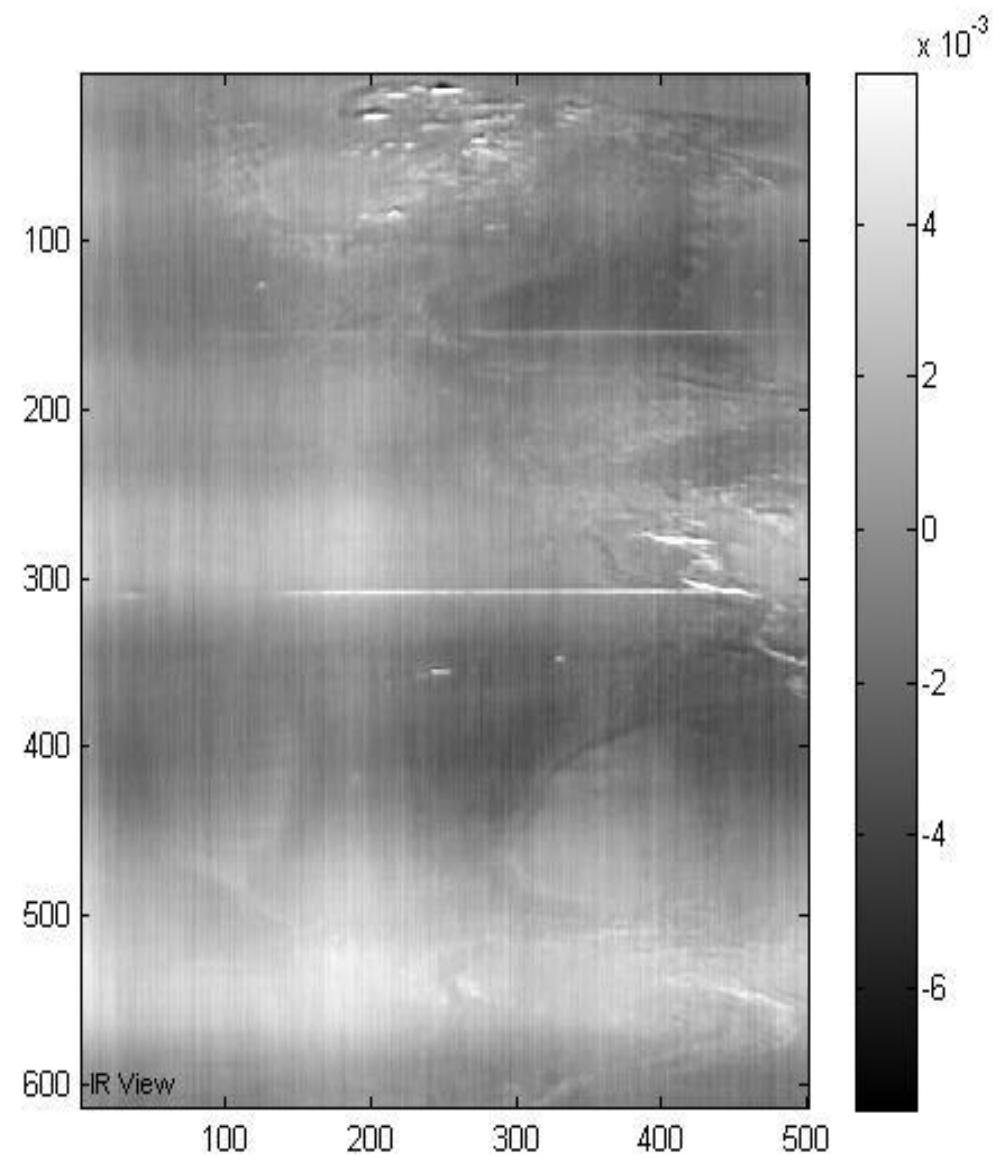

Fig. 7 specimen reconstructed with Pseudo - static reconstruction: Both $0.5 \mathrm{~mm}$ (top) and $1 \mathrm{~mm}$ (bottom) defects are visible. 


\subsection{1/qirt.2016.091}

From the experiment results, Defects of sizes width $=0.5 \mathrm{~mm}$, depth $=0.5 \mathrm{~mm}$ and width $=1 \mathrm{~mm}$ and depth $=1 \mathrm{~mm}$ are well detected (fig.5). Simulations results and the experiments match (fig.6). The time of inspection is about 20 seconds for a $20 \mathrm{cms}$ length specimen, which is correct and will be improved in future works. From the result images of the specimen line scan, the latter was reconstructed with Pseudo-static matrix reconstruction [6] and a PCT algorithm [7] is applied to enhance the result contrast. Reconstruction results are shown in figure 6. The aluminium plate is well reconstructed and the resulting image represents the aluminium plate very well with the two defects clearly seen at the right positions. The bright spots (top and middle right of the figure) seen in the resulting image represent the painting non uniformity. The specimen has been painted in black to solve the low emissivity problem. Other bright spots (under the middle defect) are due to scratches in the paint. The defect's length is the width of the part $(50 \mathrm{~mm})$. In the figure, the bright lines representing the defects do not go till the plate borders. The reason is that the coil is smaller than the specimen width. The heating is less important in specimen's edges which are "far" from the coil. The solution is to do another line scan of the specimen's part that has not been heated.

\section{Conclusion}

In this work, we presented a Robotic application of Eddy current thermography. Simulations were performed in order to determine best parameters for the experiment. Experiments were done on an aluminum plate with defects created artificially in our laboratory (line slots modelling cracks). The scanning of the part was achieved with a 6 axis robotic arm Fanuc LrMate 200iD/7L. Defects were very well detected. A reconstructed image of the specimen with the detected defects was obtained with Pseudo-static matrix reconstruction.

In future works, more complex shape specimens will be inspected and algorithms will be developed for this type of inspections. Also, we want to test of the scanning speed. Experiments will be done on specimens from the industry with real cracks.

\section{REFERENCES}

[1] P Tran-Gia, X Maldague, and L Birglen. "Crack detection limits in Eddy current thermography". The American Society for Non-destructive testing Research Symposium, (22), 2013.

[2] X Maldague. John Wiley." Theory and practice of infrared technology for non-destructive testing", 2001.

[3] I.Z Abidin, M Z Umar, M Y Yusof, and M. N Salleh. "Advantages and Applications of Eddy Current Thermography Testing for Comprehensive and Reliable Defect Assessment", 18th World Conference on Non-destructive Testing, 16-20 April, Durban, South Africa,

[4] P J Shull. "Non-destructive evaluation: theory, techniques, and applications", CRC press, 2002.

[5] H Fernandes, C Ibarra-Castanedo, $\mathrm{H}$ Zhang, and X Maldague. "Thermographic NDE of CFRP after tensile testing", Journal of NDE, 2015.

[6] C Ibarra-Castanedo, P Servais, A Ziadi, M Klein and X Maldague. "RITA - Robotized inspection by thermography and Advanced processing for the inspection of aeronautical components ", QIRT, 2014.

[7] N Rajic." Principal component thermography for flaw contrast enhancement and flaw depth characterization in composite structures", Compos. Struct., 58:521-528, 2002. 